\title{
Prospective evaluation of muscle strength and spine joint motility of patients who underwent surgery for colorectal cancer by open and laparoscopic methods
}

\author{
Iwona Głowacka-Mrotek ${ }^{1}$, Magdalena Tarkowska², Michał Jankowski ${ }^{3}$, Tomasz Nowikiewicz ${ }^{3}, Z_{\text {Zygmunt Siedlecki }}^{4}$, \\ Wojciech Hagner ${ }^{1}$, Wojciech Zegarski ${ }^{3}$ \\ ${ }^{1}$ Department of Rehabilitation, Collegium Medicum in Bydgoszcz, the Nicolaus Copernicus University in Torun, Poland \\ 2Department of Laser Therapy and Physiotherapy, Collegium Medicum in Bydgoszcz, the Nicolaus Copernicus University in Torun, Poland \\ ${ }^{3}$ Department of Surgical Oncology, Collegium Medicum in Bydgoszcz, the Nicolaus Copernicus University in Torun, Poland \\ ${ }^{4}$ Department of Neurosurgery, Neurotraumatology and Paediatric Neurosurgery, Collegium Medicum in Bydgoszcz, \\ the Nicolaus Copernicus University in Torun, Poland
}

Videosurgery Miniinv 2020; 15 (1): 49-57 DOI: https://doi.org/10.5114/wiitm.2019.84762

\begin{abstract}
Introduction: The current trend in oncological surgery is to minimize its degree of invasiveness while maintaining a satisfactory survival rate. Surgical treatments within the large intestine are applied through traditional open surgery (OS) or laparoscopic surgery (LS).

Aim: The purpose of this nonrandomized, prospective, single-centered clinical examination was to compare motility within the spine joints and evaluate abdominal muscle strength of patients who underwent LS or OS for colorectal cancer.

Material and methods: Seventy-two patients were included in the study. Open surgery was applied to 35 patients and LS was applied to 37 patients. Motility range of the thoracic and lumbar spine, muscle strength of abdominal muscles, and pain evaluation by the Visual Analogue Scale (VAS) of the studied group were evaluated twice (on the day of admission to the ward and on the fifth day after the surgery).

Results: Both types of surgical intervention resulted in a decrease of the rectus abdominis and abdominal oblique muscle strength as well as a decrease of the range of thoracic and lumbar spine joint motility $(p<0.001)$. In the first research period, no statistically significant differences of tested parameters between the groups were found. In the second period, patients who underwent $L S$ achieved better results within the extension of lumbar spine section $(p=0.0339)$, rectus abdominis strength $(p=0.0105)$, and left abdominal oblique muscles $(p=0.004)$.

Conclusions: Both types of surgical intervention (LS and OS) result in reduction of spine joint motility range and abdominal muscle strength. Laparoscopic surgery disrupts the spine joint motility and abdominal muscle strength to a lesser extent than OS.
\end{abstract}

Key words: laparoscopic surgery, open surgery, recovery, abdominal muscle strength.

\section{Introduction}

As colorectal cancer becomes more common, it becomes more challenging for contemporary oncol- ogy. The forecasted frequency of this illness for 2030 is 2.2 million patients and the highest probability of falling sick is connected with living in Westernized

\footnotetext{
Address for correspondence

Iwona Głowacka-Mrotek PhD, Department of Rehabilitation, Collegium Medicum, the Nicolaus Copernicus University,

9 Curie-Skłodowskiej St, 85-094 Bydgoszcz, Poland, phone: +48 7912253 18, e-mail: iwona.glowacka@cm.umk.pl
} 
countries [1]. Among the many methods of treating neoplastic diseases (chemotherapy, radiotherapy, hormone therapy), surgery still remains the only radical therapeutic option [2]. Since their introduction in the 1990s, laparoscopic methods have played increasingly significant roles among surgical techniques. Randomized clinical examinations and meta-analyses show that colorectal cancer surgery using laparoscopic techniques results in less blood loss during surgery, shorter surgery time, faster peristalsis return, faster oral nutrition implementation, less pain after surgery, and a better cosmetic effect [3]. Randomized clinical examinations show that the desired results of oncological treatment (overall survival, disease-free survival) using laparoscopic methods are comparable with the OS methods [1-5]. Laparoscopy is an alternative to OS for treating even advanced tumors [3].

During surgery, both OS and LS methods break the abdominal muscles' continuity. A patient must restrict physical activity in the perioperative period [6], and the postoperative wound, which is the source of pain, limits the motility range at the spine joint area. There is an abundance of research confirming the benefits of laparoscopic surgery [1-8]. Most of these studies are based on observations of time of survival, measurements of lost blood, hospitalization time, and duration of surgery [1-8]. Both intervention types (LS and OS) interfere with the human organism. The treated area (external part of the skin and abdominal muscles) is violated and during the perioperative and postoperative periods, the patient is exposed to stress [9]. Postoperative complications after laparoscopic colorectal cancer surgery have a significant impact on long-term outcomes [10]. In the postoperative period, patients limit their physical activity, which can lead to spine pain later. There is a lack of research evaluating the impact of surgical methods (OS, LS) on muscle strength in areas of the body that underwent surgery (rectus abdominis and abdominal oblique muscles) and the impact of the surgery on motility range restrictions in the treatment area (thoracic and lumbar spine). In our study we present short-term outcomes. Shortterm outcomes are a very important indicator. Generally patients 5 days after surgery they should be fed orally and be ready to leave the hospital [4-7].

\section{Aim}

The aim of the study was to evaluate the impact of both treatments (LS and OS) on abdominal mus- cle strength and the spine motility range of patients who underwent surgery for colorectal cancer.

\section{Material and methods}

This prospective study was conducted on the basis of Collegium Medicum of the Nicolaus Copernicus University in Torun Bioethical Committee consent no. 473/2016. Research began on September 1 , 2016, and was concluded on March 31, 2017. Patients referred to the Oncological Surgery Clinic and Department were included in the study because they needed colorectal cancer surgery (rectal carcinoma). One hundred three patients were initially included in the study. After taking into account the inclusion and exclusion criteria, the analyzed group was narrowed to 72 people. Figure 1 presents the study design in detail. Thirty-five patients underwent LS and 37 underwent OS. Preoperative diagnostics of the studied group included basic examination, anesthetic evaluation, colonoscopy, X-ray chest examination, ultrasound examination, and computed tomography (CT) examination of the abdominal cavity. Patients' intestines were prepared mechanically for surgery and each received general anesthesia during surgery. After surgery, every patient was monitored in the postoperative room and received analgesic treatment. The extent of resection was much the same for laparoscopic and open surgery. The specimen was removed through a midline mini-laparotomy wound - in the place used for a 10-mm camera port (placed through a subumbilical midline incision). The incision length was decided according to tumor size. The average incision length was $4.7 \mathrm{~cm}$ (range: $4.5-5.2 \mathrm{~cm}$ ) for all operated patients.

All laparoscopic procedures were performed by the same laparoscopic surgeon, who had performed more than 200 such procedures. The open procedures were performed by four surgeons from one team.

Inclusion criteria were: consent to participate in the study, diagnosis of colorectal cancer, chose elective surgery, was 18 years old or older, was a candidate for curative resection involving only the large intestine, qualified for the OS or LS, ASA I-III, cancer severity I-III (WHO), Karnofsky score of 80-100 points.

Exclusion criteria were: previous surgery for colorectal cancer, diagnosis of stage IV of cancer severity, incomplete medical documentation, stoma 


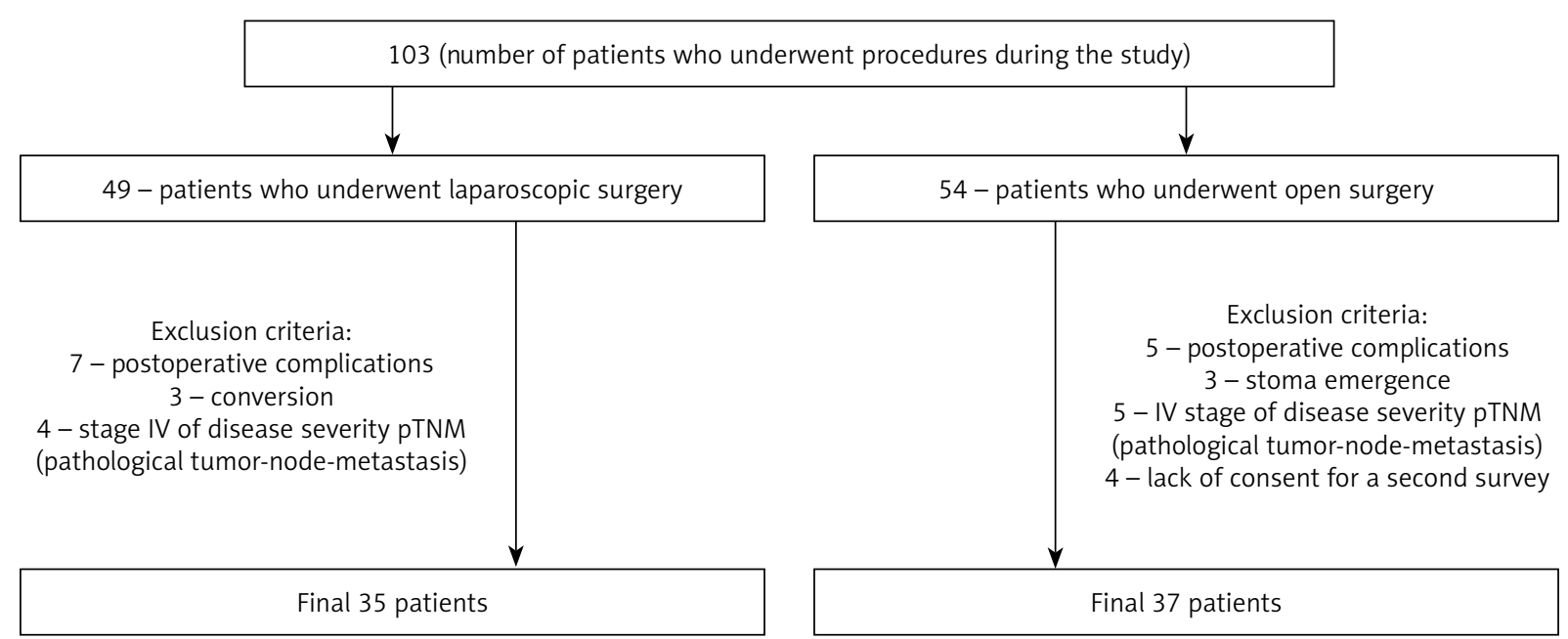

Figure 1. Scheme describing the exclusion of patients from the study

created at surgery, multiple resection procedures, and patients for whom the conversion was applied during surgery.

In order to evaluate the motility and mobility disorders in the treated area, the patient's motility range in the thoracic and lumbar segments of the spine and rectus abdominis and abdominal oblique muscle strength was evaluated. Spine motility was measured using tailor's tape marked in $0.5 \mathrm{~cm}$ increments. Spine motility was measured with the patient in a standing, free position. The following parameters of spine motility were evaluated:

- flexion of the thoracic spine (the distance between spinous process of the first thoracic vertebra (Th1) and the last thoracic vertebra (Th12)) was measured from PW (Starting Position). The next measurement was made as the patient performed a maximal bend forward with straight knees in the PK (Final Position). The difference between PK and PW was determined to be the motility range of the thoracic spine flexion;

- lumbar spine flexion (the distance between spinous process of the first lumbar vertebra and the spinous process of the fifth lumbar vertebra) was measured at PW. The next measurement was made as the patient bent forward with straight knees (PK). The difference between PK and PW defined the motility range;

- complete spine bend (bending forward). The distance from the protuberantia occipitalis externa to the median sacral crest aitchbone was measured at PW. The next measurement was made when the patient performed a complete bend forward with straight knees. The difference between PK and PW defined the motility range;

- lumbar spine extension (the distance between the end of the breastbone xiphoid process and the pubis) was measured. The next measurement was made when the patient performed a bend backward with straight knees (PK). The difference between PK and PW was assumed to be the motility range;

- lateral thoracolumbar spine flexion (the distance between the top of the axillary fossa and the wing of the ilium) was measured (PW). The next measurement was made when the patient performed a maximal lateral bend (PK). The difference between PK and PW defined the motility range. This measurement was made for both right and left sides;

- thoracolumbar spine twist (the distance from the end of the xiphoid process to the anterior superior iliac spine) was measured. The next measurement was made when the patient performed a waist twist in the opposite direction. The difference between PK and PW defined the motility range. This measurement was made for both right and left sides.

Additionally, abdominis muscle strength was evaluated using the muscle strength measuring apparatus MICROFET 2 produced by TECHNOMEX. The following parameters were evaluated:

- rectus abdominis muscle strength was evaluated with the patient in a prone position, lying on the back with legs bent at the hip and knee joints. When the patient was instructed to bend forward, 
the apparatus was placed on the patient's rectus abdominis above the navel while performing the action. The highest score displayed by the apparatus was recorded;

- abdominal oblique muscle strength was evaluated while the patient was lying on the back with lower limbs bent at the hip and knee joints. The patient was instructed to touch the knee of the opposite side with the elbow as strength of the abdominal muscles was evaluated. The measurement was made for both right and left sides. The highest score was documented.

Evaluation of the range of spine motion and abdominal muscle strength was performed twice: on the day of admission to the ward (before starting the mechanical intestines cleansing) and on the fifth day after surgery. The fifth day after surgery was chosen for evaluation due to our assumption that all patients would require a hospital stay, independent of surgical intervention type. Analysis of the average time a patient spent in the hospital after surgery would include a fifth day for all study participants.

On the fifth day after the surgery, the level of patient pain was evaluated using the Visual Analogue Scale (VAS). Each patient was instructed to identify their pain level on a paper card displaying a 0-10 scale where 0 meant no pain and 10 meant the most intense pain the patient could imagine. Results were recorded and compared for both the LS and OS groups.

\section{Statistical analysis}

Statistical studies were conducted using the statistical package PQStat v1.6.6.202. Demographic and clinical data were compared between the groups using Student's $t$-test. Scales were quantitative and rarely deviated from normal distribution as verified by the Shapiro-Wilk test (age, body weight, height, and body mass index (BMI)). When deviation was present, additional analysis using the Mann-Whitney $U$ test (hospitalization time) was performed. Analysis of quantitative scales used the $\chi^{2}$ dependence test.

Motility range results of spine joints and $a b$ dominal muscles were subjected to non-parametric analysis. The study groups' results were compared by the Mann-Whitney $U$ test. Comparison of before- and after-treatment results used the Wilcoxon signed-ranked test. The VAS scale results in both groups were compared by the Mann-Whitney $U$ test.
The level of $p<0.05$ was considered significant and $p<0.01$ was considered highly significant.

\section{Results}

The demographic and clinical pathological characteristics for all 72 patients are listed in Table I. The laparoscopic and open groups were similar in terms of age, gender, height, weight, body mass index (BMI), surgical procedure, severity of cancer, and preoperative treatment. Length of hospital stay differed significantly between the two groups (LS and OS), with the OS group hospitalized longer $(p=0.0451)$. Spine joint motility and rectus abdominis and abdominal oblique muscle strength of the studied groups were evaluated on the day of admission and on the fifth day after surgery. A subjective evaluation of pain defined by the VAS scale was also conducted. Results are shown in Table II. In both groups (LS and OS), the decrease in motility ranges in spine joints was observed to be highly statistically significant high $(p<0.001)$. Comparison of dependency between the LS and OS groups did not show any statistically significant differences on the first date of measurement. Statistically significant differences were noted, however, in terms of motility range, lumbar spine flexion $(p=0.0339)$, rectus abdominis muscle strength $(p=0.0105)$, and left abdominal oblique muscle strength $(p=0.004)$ during second evaluations. Patients in the LS group achieved higher results. On the second day of the study, patients subjectively evaluated their pain. Lower scores and less pain were noted in the LS group; the results were statistically significant $(p=0.0002)$. Results are shown in Table III.

\section{Discussion}

The purpose of our research was to evaluate the motility range within the spine joints, abdominal muscle strength, and pain levels of patients who underwent laparoscopic and open surgery for colorectal cancer. The measurements were made twice: on the day of admission to the surgical ward and again on the fifth day after surgery. Results of our research reveal a highly statistically significant decrease in spine joint motility range in both groups. Comparison of the dependency between the groups (OS and LS) showed that patients from the LS group obtained significantly higher results within the lumbar spine flexion range and left abdominal oblique muscle 
Table I. Demographic and clinical data

\begin{tabular}{|c|c|c|c|}
\hline Variable & $\operatorname{LS}(n=35)$ & OS $(n=37)$ & $P$-value \\
\hline $\begin{array}{l}\text { Age, mean (range) } \\
\text { SD }\end{array}$ & $\begin{array}{c}64.89(35-87) \\
12.31\end{array}$ & $\begin{array}{c}65.40(35-84) \\
10.57\end{array}$ & 0.8536 \\
\hline Gender: & & & 0.4793 \\
\hline Female & 17 & 19 & \\
\hline Male & 20 & 16 & \\
\hline $\begin{array}{l}\text { Body weight, mean (range) } \\
\text { SD }\end{array}$ & $\begin{array}{c}76.84(52-104) \\
12.28\end{array}$ & $\begin{array}{c}77.41(56-112) \\
14.77\end{array}$ & 0.8587 \\
\hline $\begin{array}{l}\text { Height, mean (range) } \\
\text { SD }\end{array}$ & $\begin{array}{c}1.73(1.7-1.8) \\
0.09\end{array}$ & $\begin{array}{c}1.69(1.7-1.85) \\
0.09\end{array}$ & 0.0597 \\
\hline $\begin{array}{l}\text { BMI, mean (range) } \\
\text { SD }\end{array}$ & $\begin{array}{c}25.78(26-29.6) \\
4.00\end{array}$ & $\begin{array}{c}27.10(27.1-29.4) \\
4.52\end{array}$ & 0.1911 \\
\hline Surgical treatment type: & & & 0.4070 \\
\hline Anterior resection of the rectum & $16(44.44 \%)$ & $19(54.29 \%)$ & \\
\hline Colon resection: & $20(55.56 \%)$ & $16(45.71 \%)$ & \\
\hline Right hemicolectomy & 8 & 7 & \\
\hline Left hemicolectomy & 4 & 3 & \\
\hline Ssigmoid resection & 8 & 6 & \\
\hline Duration in surgery [min] $]^{*}$ : & & & $<0.0001$ \\
\hline Rectal resection & $145(95-175)$ & $123(80-167)$ & \\
\hline Colon resection & $125(55-155)$ & $105(45-135)$ & \\
\hline Clinical stage (pTNM): & & & 0.0537 \\
\hline I & $3(8.10 \%)$ & $8(22.86 \%)$ & \\
\hline$\|$ & $16(43.24 \%)$ & $7(20.00 \%)$ & \\
\hline III & $18(48.65 \%)$ & $20(57.14 \%)$ & \\
\hline Preoperative treatment: & & & $<0.0001$ \\
\hline $\mathrm{CHTH}$ & $0(0 \%)$ & $3(8.57 \%)$ & \\
\hline RTH & $12(0 \%)$ & $14(40 \%)$ & \\
\hline $\mathrm{CHTH}+\mathrm{RTH}$ & $1(10.81 \%)$ & $1(2.86 \%)$ & \\
\hline Deficiency & $22(89.19 \%)$ & $17(48.57 \%)$ & \\
\hline Postoperative treatment: & & & 0.6324 \\
\hline $\mathrm{CHTH}$ & $19(51.35 \%)$ & $16(45.71 \%)$ & \\
\hline RTH & $0(0 \%)$ & $0(0 \%)$ & \\
\hline $\mathrm{CHTH}+\mathrm{RTH}$ & $0(0 \%)$ & $0(0 \%)$ & \\
\hline Deficiency & $18(48.65 \%)$ & $19(54.29 \%)$ & \\
\hline $\begin{array}{l}\text { Hospitalization time, median } \\
\text { mix.-max. }\end{array}$ & $5(6-13)$ & $7(6-20)$ & 0.0451 \\
\hline
\end{tabular}

$n$-number of patients, LS - patients who underwent laparoscopic surgery, OS - patients who underwent open survey, BMI-body mass index, SD - standard deviation. *Time from first incision to skin closure, $T$ - tumor, $N$ - nodes, $M$ - metastasis, min.-max. - minimum-maximum, CHTH - chemotherapy, RTH roentgenotherapy, $p$-statistical significance level indicator. 
Table II. Comparison of spine joint motility, rectus abdominal and abdominal oblique muscle strength, pain evaluation on the VAS scale of the studied groups (OS, LS), and evaluation of dependency between the groups

\begin{tabular}{|c|c|c|c|c|c|c|c|c|c|}
\hline \multirow[t]{2}{*}{ Variable } & & \multicolumn{3}{|c|}{$\operatorname{LS}(n=35)$} & \multicolumn{3}{|c|}{ OS $(n=37)$} & \multicolumn{2}{|c|}{ Mann-Whitney $U$ test } \\
\hline & & Mean & $\begin{array}{l}\text { Standard } \\
\text { deviation }\end{array}$ & Median & Mean & $\begin{array}{l}\text { Standard } \\
\text { deviation }\end{array}$ & Median & $Z$ & $P$-value \\
\hline \multirow{2}{*}{$\begin{array}{l}\text { Thoracic spine } \\
\text { flexion }\end{array}$} & I & 2.38 & 0.76 & 2 & 2.36 & 1.71 & 2 & 1.5747 & 0.1153 \\
\hline & ॥ & 1.35 & 0.63 & 1 & 1.06 & 0.75 & 1 & 1.8943 & 0.0582 \\
\hline Wilcoxon's test & & \multicolumn{3}{|c|}{$Z=4.7318, p<0.0001$} & \multicolumn{3}{|c|}{$Z=3.563, p=0.0004$} & & \\
\hline \multirow{2}{*}{$\begin{array}{l}\text { Complete spine } \\
\text { bend }\end{array}$} & 1 & 9.65 & 3.95 & 9 & 8.97 & 2.87 & 8 & 0.9602 & 0.3369 \\
\hline & ॥ & 5.11 & 2.42 & 5 & 4.39 & 2.16 & 4 & 1.3028 & 0.1927 \\
\hline Wilcoxon's test & & \multicolumn{3}{|c|}{$Z=4.7318, p<0.0001$} & \multicolumn{3}{|c|}{$Z=5.0143, p<0.0001$} & & \\
\hline \multirow{2}{*}{$\begin{array}{l}\text { Lumbar spine } \\
\text { extension }\end{array}$} & I & 4.16 & 2.21 & 4 & 4.11 & 1.89 & 3.5 & 0.028 & 0.9776 \\
\hline & II & 2.62 & 2.24 & 2 & 1.78 & 1.53 & 2 & 1.6591 & 0.0971 \\
\hline Wilcoxon's test & & \multicolumn{3}{|c|}{$Z=3.4298, p=0.0006$} & \multicolumn{3}{|c|}{$Z=4.5316, p<0.0001$} & & \\
\hline \multirow{2}{*}{$\begin{array}{l}\text { Lateral thora- } \\
\text { columbar spine } \\
\text { flexion, right side }\end{array}$} & 1 & 5.86 & 2.71 & 5 & 5.28 & 1.75 & 5 & 0.7468 & 0.4552 \\
\hline & ॥ & 3.51 & 1.98 & 3 & 2.58 & 1.56 & 3 & 1.9519 & 0.0509 \\
\hline Wilcoxon's test & & \multicolumn{3}{|c|}{$Z=4.7210, p<0.0001$} & \multicolumn{3}{|c|}{$Z=4.5525, p<0.0001$} & & \\
\hline \multirow{2}{*}{$\begin{array}{l}\text { Lateral thora- } \\
\text { columbar spine } \\
\text { flexion, left side }\end{array}$} & 1 & 5.73 & 2.88 & 5 & 5.31 & 1.83 & 5 & 0.1564 & 0.8757 \\
\hline & ॥ & 1.89 & 1.54 & 2 & 1.72 & 1.37 & 1 & 0.7022 & 0.4826 \\
\hline Wilcoxon's test & & \multicolumn{3}{|c|}{$Z=4.9929, p<0.0001$} & \multicolumn{3}{|c|}{$Z=4.9944, p<0.0001$} & & \\
\hline \multirow{2}{*}{$\begin{array}{l}\text { Lumbar spine } \\
\text { flexion }\end{array}$} & 1 & 4.92 & 1.75 & 5 & 4.50 & 2.21 & 4 & 1.449 & 0.1473 \\
\hline & II & 3.27 & 2.32 & 3 & 2.08 & 1.25 & 2 & 2.1209 & 0.0339 \\
\hline Wilcoxon's test & & \multicolumn{3}{|c|}{$Z=3.7610, p=0.0002$} & \multicolumn{3}{|c|}{$Z=4.5556, p<0.0001$} & & \\
\hline \multirow{2}{*}{$\begin{array}{l}\text { Rectus abdominis } \\
\text { muscle strength }\end{array}$} & 1 & 26.86 & 11.80 & 24.4 & 22.91 & 7.85 & 22.05 & 1.4349 & 0.1513 \\
\hline & ॥ & 17.80 & 6.94 & 17.6 & 14.01 & 6.66 & 12.3 & 2.5603 & 0.0105 \\
\hline Wilcoxon's test & & \multicolumn{3}{|c|}{$Z=5.2956, p<0.0001$} & \multicolumn{3}{|c|}{$Z=4.6268, p<0.0001$} & & \\
\hline \multirow{2}{*}{$\begin{array}{l}\text { Abdominal } \\
\text { oblique muscle } \\
\text { strength, right } \\
\text { side }\end{array}$} & I & 25.86 & 12.01 & 21 & 21.34 & 7.84 & 20.2 & 1.2251 & 0.2205 \\
\hline & ॥ & 16.06 & 8.64 & 14.5 & 14.31 & 6.63 & 13.4 & 0.5077 & 0.6117 \\
\hline Wilcoxon's test & & \multicolumn{3}{|c|}{$Z=4.6089, p<0.0001$} & \multicolumn{3}{|c|}{$Z=4.2015, p<0.0001$} & & \\
\hline \multirow{2}{*}{$\begin{array}{l}\text { Abdominal } \\
\text { oblique muscle } \\
\text { strength, left side }\end{array}$} & I & 27.55 & 13.60 & 22.6 & 21.60 & 7.42 & 19.1 & 1.8267 & 0.0677 \\
\hline & $\|$ & 18.94 & 9.15 & 17.05 & 13.18 & 5.89 & 12.85 & 2.8783 & 0.004 \\
\hline Wilcoxon's test & & \multicolumn{3}{|c|}{$Z=4.4854, p<0.0001$} & & $8469, p<0$ & 001 & & \\
\hline
\end{tabular}

$n$ - number of patients, LS - patients who underwent laparoscopic surgery, OS - patients who underwent open surgery, I - study before the surgery, II - study on the fifth day after the surgery, $p$ - statistical significance level indicator.

Table III. VAS (Visual Analogue Scale) results (LS vs. OS)

\begin{tabular}{|lcccc|}
\hline Group & Mean & Standard deviation & Median & Mann-Whitney $U$ test \\
\hline LS $(n=35)$ & 3.65 & 1.46 & 4 & $Z=3.7388$ \\
OS $(n=37)$ & 5.17 & 1.72 & 5 & $p=0.0002$ \\
\hline
\end{tabular}

$n$-number of patients, LS - patients who underwent laparoscopic surgery, OS - patients who underwent open surgery. 
strength, as well as rectus abdominis strength. Patients from the LS group also reported statistically lower pain levels, assessed by the VAS scale.

Discussion of the topic of role and meaning of laparoscopic procedures in treatment of euplastic diseases has been ongoing for quite some time. Currently, laparoscopic surgery is an approved way of treating patients suffering from colon cancer; it has similar oncological treatment results (overall survival rate, disease-free survival) as classical treatments. On the basis of publicized meta-analyses and randomized studies, it is believed that laparoscopic methods benefit the patient by less blood loss and faster intestinal peristalsis return [1-8]. Our research is the first to evaluate the impact of these treatments (LS and OS) on spine motility and rectus abdominis and abdominal oblique muscle strength. Both types of surgical intervention violate the continuity of the outer and subcutaneous skin layers and muscle tissue as well as the internal organs. The results of our research reveal that both groups (LS and OS) experienced a statistically significant decrease in motility range of the lumbar and thoracic spine joint sections, as well as complete spine extension and statistically significant muscle strength reduction. Postoperative patients often assume analgesic body positions, stay in bed, and limit physical activity in the first days following surgery. Preparation for surgery was the same regardless of its type. Research shows that in the preoperative period, patients preparing for surgery experienced sleeping disorders and limited their physical activities [6, 9-12]. After successful surgery, the incidence of sleeplessness decreased $[13,14]$. Research conducted by Ekblom-Bak et al. and Onerup et al. provides interesting conclusions: the authors found that increasing appropriate physical activities in the postoperative period can result in shorter convalescence times after surgery $[15,16]$. This type of solution is not realistically possible, however, to implement in medium-developed countries. Research shows that patient mobility increases after release from hospitalization. Other authors' studies [1-8] and our research indicate shorter hospitalization times when laparoscopic methods are used.

In our study we present short-term results.

Patients received the same standard preoperative (arrangements made for treatment and intestine preparation) and postoperative management. Other authors' research shows that a faster return to fitness was shown by patients who were treated with laparoscopy and fast-track rehabilitation (FTR) or enhanced recovery after surgery (ERAS) protocols $[17,18]$. The postoperative procedure our center follows includes ERAS protocol elements. Postoperative hospitalization time of the analyzed patients, on average, did not differ excessively from the data of most recent publications [19, 20]. Other authors' research shows that early closure of protective ileostomy is safe should be implemented as part of the ERAS protocol for rectal cancer patients [21].

The strong point of our research is the fact that it was conducted prospectively. All patients experienced identical preoperative examinations followed by re-evaluation of spine motility range and rectus abdominis and abdominal oblique muscle strength on the same timeline after surgery. Our research was conducted using objective methods, spine motility measurements using tailor's tape, and the appropriate muscle-strength measuring tool.

Our research showed that after 5 days from surgery, patients who underwent LS achieved statistically significantly higher results within lumbar spine flexion motion than those in the OS group. Thoracic spine flexion motion and right thoracolumbar spine lateral flexion were just within the zone of statistical significance $(p=0.05)$. During the second round of evaluations, statistically significant muscle strength measurement differences were shown. Patients undergoing laparoscopic surgery had statistically significantly higher abdominal muscle $(p=0.0105)$ and left abdominal oblique muscle strength $(p=0.004)$. Abdominal muscles fulfill an important function in the organism: they are stabilizers. Spine motility range limitation can be the cause of spine pain for patients who have surgery $[22,23]$. The other problem of patients who underwent colorectal cancer surgery is the postoperative scar. This scar can be a source of pain and motility limitation if it is not mobilized appropriately. Our research reveals how important it is for early postoperative patients to begin regaining mobilization and to work on spine joint motility. This revelation is true regardless of the type of surgical intervention, LS or OS. The aim of our research was pain evaluation of patients who underwent laparoscopic and open procedures. According to our research, patients who had LS described lower pain levels on the fifth day after surgery than patients who underwent OS. Other researchers describe similar results regarding patient perception of post-surgical pain [24]. 
Our study presents short-term results; we made our measurements before surgery and 5 days after surgery. Short-term results are important indicators of feasibility and safety of laparoscopic and open surgery $[25,26]$. In our study operating times were significantly longer in the laparoscopic surgery group than in the open surgery group. Similar findings were obtained in a previous study [27].

Our research has limitations typical of a single-centered non-randomized analysis. The present study has certain limitations, mainly consisting in a quite small population of patients included in the analysis. According to other researchers, however, execution of a randomized controlled trial within the studied group was not possible because patients were recruited for the study consecutively, upon admission. As a result, only short-term outcomes were analyzed in this study. It could be worthwhile to conduct an evaluation of abdominal muscle strength and measurement of range of motion during a longer-term postoperative evaluation. Study of longer-term postoperative results may be the only way to discover how the abdominal muscle strength and spine joint motility change following OS and LS. The third limitation is due to the fact that the study group is very heterogeneous. Carrying out the study on a more homogeneous group will be more beneficial.

\section{Conclusions}

Our research shows that, in both groups (LS and OS) on the fifth day after surgery, a statistically significant reduction of thoracic and lumbar spine motility range occurred. This fifth-day evaluation also revealed that a reduction of rectus abdominis and abdominal oblique muscle strength levels occurred. Differences in measurement results were lower in the LS group. Laparoscopic procedures offer patients a chance for faster recovery. Our research suggests that patients should be recommended for a rehabilitation program focusing on improvement of spine joint motility range and abdominal muscle enhancement, regardless of which surgical procedure (LS or OS) is used.

\section{Acknowledgments}

Grant support for the study was received from the Ministry of Science and Higher Education of Poland No. MN-6/WNoZ/2016. The financial sponsors had no role in study design, data collection and analysis, or preparation of this article. The authors have no financial interest in any of the products or devices mentioned in this article.

\section{Conflict of interest}

The authors declare no conflict of interest.

\section{References}

1. Arnold M Sierra, Laversanne M, et al. Global patterns and trends in colorectal cancer incidence and mortality. Gut 2017; 66: 683-91.

2. Liu C, Liu J, Zhang S. Laparoscopic versus conventional open surgery for immune function in patients with colorectal cancer. Int J Colorectal Dis 2011; 26: 1375-85.

3. Braga M, Frasson M, Vignali A, et al. Laparoscopic vs. open colectomy in cancer patients: long-term complications, quality of life, and survival. Dis Colon Rectum 2005; 48: 2217-23.

4. Nienhüser H, Heger P, Schmitz R, et al. Short- and long-term oncological outcome after rectal cancer surgery: a systematic review and meta-analyzis comparing open versus laparoscopic rectal cancer surgery. J Gastrointest Surg 2018; 22: 1418-33.

5. Veldkamp R, Kuhry E, Hop WC, et al. Laparoscopic surgery versus open surgery for colon cancer: short-term outcomes of a randomized trial. Lancet Oncol 2005; 6: 477-84.

6. Bonjer HJ, Deijen CL, Abis GA, et al. COLOR II Study Group. A randomized trial of laparoscopic versus open surgery for rectal cancer. N Engl J Med 2015; 372: 1324-32.

7. Yilmaz M, Sezer H, Gürler H, Bekar M. Predictors of preoperative anxiety in surgical inpatients. J Clin Nurs 2011; 21: 956-64.

8. Reza MM, Blasco JA, Andradas E, et al. Systematic review of laparoscopic versus open surgery for colorectal cancer. Br J Surg 2006; 93: 921-8.

9. Tjandra JJ, Chan MK. Systematic review on the short-term outcome of laparoscopic resection for colon and rectosigmoid cancer. Colorectal Dis 2006; 8: 375-88.

10. Nowakowski M, Pisarska M, Rubinkiewicz M, et al. Postoperative complications are associated with worse survival after laparoscopic surgery for non-metastatic colorectal cancer - interim analysis of 3-year overall survival. Videosurgery Miniinv 2018; 13: 326-32.

11. Santos M, Martinsose CA, Oliveria LM. Anxiety, depression and stress in the preoperative surgical patient. Rev Enferm Ref 2014; 4: 7-15.

12. Dronkers JJ, Chorus AM, van Meeteren NL, Hopman-Rock M. The association of pre-operative physical fitness and physical activity with outcome after scheduled major abdominal surgery. Anaesthesia 2013; 68: 67-73.

13. Taşdemir A, Erakgün A, Nuri Deniz M, Certuğ A. Comparison of preoperative and postoperative anxiety levels with statetrait anxiety inventory test in preoperatively informed patients. Turkish J Anesth Reanim 2013; 41: 44-9.

14. Yılmaz Y, Durmuş K, Yılmaz Inan F. The effects of preoperative and postoperative anxiety on postoperative pain and analgesic consumption in septoplasty. Dicle Med J 2014; 41: 288-93.

15. Ekblom-Bak E, Olsson G, Ekblom O, et al. The daily movement pattern and fulfilment of physical activity recommendations in 
Swedish middle-aged adults: the SCAPIS pilot study. PLoS One 2015; 10: e0126336.

16. Onerup A, Angenete E, Bock D, et al. The effect of pre- and post-operative physical activity on recovery after colorectal cancer surgery (PHYSSURG-C): study protocol for a randomised controlled trial. Trials 2017; 18: 212.

17. Vlug MS, Wind J, Hollmann MW, et al. Laparoscopy in combination with fast track multimodal management is the best perioperative strategy in patients undergoing colonic surgery. A randomized clinical trial (LAFA-study). Ann Surg 2011; 254: 868-75.

18. Esteban F, Cerdan FJ, Garcia-Alonso M, et al. A multicentre com parison of a fast track or conventional postoperative protocol following laparoscopic or open elective surgery for colorectal cancer surgery. Colorectal Disease 2013; 16: 134-40.

19. Richardson CA, Snijders CJ, Hides JA, et al. The relation between the transverses abdominis muscles, sacroiliac joint mechanics, and low back pain. Spine 2002; 15: 399-405.

20. Hodges PW, Richardson CA. Inefficient muscular stabilization of the lumbar spine associated with low back pain. Spine 1996; 21: 2640-50.

21. Kłęk S, Pisarska M, Milian-Ciesielska K, et al. Early closure of the protective ileostomy after rectal resection should become part of the Enhanced Recovery After Surgery (ERAS) protocol: a randomized, prospective, two-center clinical trial. Videosurgery Miniinv 2018; 13: 435-41.

22. Wahl TS, Goss LE, Morris MS, et al. Enhanced recovery after surgery (ERAS) eliminates racial disparities in postoperative length of stay after colorectal surgery. Ann Surg 2018; 268: 1026-35.

23. Zhuang CL, Ye XZ, Zhang XD, et al. Enhanced recovery after surgery programs versus traditional care for colorectal surgery: a meta-analysis of randomized controlled trials. Dis Colon Rectum 2013; 56: 667-78.

24. Bédard D, Purden MA, Sauvé-Larose N, et al. The pain experience in post surgical patients following the implementation of an evidence-based approach. Pain Manag Nurs 2006; 7: 80-92.

25. Zeng WG, Zhou ZX, Hou HR, et al. Outcomes of laparoscopic versus openresection for rectal cancer in elderly patients. JSR 2015; 193: 613-8.

26. Vallribera Valls F, Landi F, Espin Basany E, et al. Laparoscopy-as sisted versus opencolectomy for treatment of colon cancer in the elderly: morbidity and mortality outcomes in 545 patients. Surg Endosc 2014; 28: 3373-8.

27. Lian L, Kalady M, Geisler D, Kiran RP. Laparoscopic colectomy is safe and leads to a significantly shorter hospital stay for octogenarians. Surg Endosc 2010; 24: 2039-43.

Received: 2.01.2019, accepted: 1.04.2019. 